\title{
Optimal Off-Exchange Execution with Closing Price
}

\author{
Seiya Kuno', Masamitsu Ohnishi'1,2, Peilu Shimizu* \\ ${ }^{1}$ Center for Mathematical Modeling and Data Science, Osaka University, Osaka, Japan \\ ${ }^{2}$ Graduate School of Economics, Osaka University, Osaka, Japan \\ Email: kuno@sigmath.es.osaka-u.ac.jp, ohnishi@econ.osaka-u.ac.jp
}

How to cite this paper: Kuno, S., Ohnishi, M. and Shimizu, P. (2017) Optimal OffExchange Execution with Closing Price. Journal of Mathematical Finance, 7, 54-64. https://doi.org/10.4236/jmf.2017.71003

Received: October 19, 2016

Accepted: January 16, 2017

Published: January 19, 2017

Copyright $\odot 2017$ by authors and Scientific Research Publishing Inc. This work is licensed under the Creative Commons Attribution International License (CC BY 4.0).

http://creativecommons.org/licenses/by/4.0/

\begin{abstract}
The purpose of this paper is to examine whether the closing price guaranteed execution is possible contract, and if possible, how an institutional investor who affects the security price allocates execution volumes to both traditional trading and off-exchange (over-the-counter, OTC) trading venues. With a generalized price model at the traditional venue which considers the permanent impact effect explicitly, we derive an optimal execution strategy in the traditional trading venue and the allocation of the order volume to both venues in the framework of dynamic programming. By proving that an optimal execution strategy is in the static class, we further show that the closing price guaranteed contract may be established and the trading volume at the time of agreement of the contract can be controlled. Moreover, by some numerical examples, we illustrate a possibility for the institutional investor to manipulate the market in order to seek a profit under some trading situation.
\end{abstract}

\section{Keywords}

Optimal Execution, Algorithmic Trading, Off-Exchange Trading, Closing Price Guaranteed Execution

\section{Introduction}

In recent years, as the increase of trading opportunities as the stock trading "venue," it has also been diversified the ways of trading for the institutional investors who execute a large amount of their order. An institutional investor, referred to as the large trader, has to take into account of market impacts because of the liquidity of supply and demand at the stock exchange. That is to say, if the large trader wants to purchase a large amount of her order of a specific stock at the stock exchange, it is hard for her to find counter-sellers who satisfy her or${ }^{\star}$ Ms. Shimizu, P. is now a housewife, and does not have any other occupation. 
ders from unspecified number of trading participants. Thus, she must consider the risk of price shift up. On the contrary, if someone who wants to sell a large amount, it is necessary to take into account the risk of price down. This type of problem has been tackled by many researchers and practitioners so far. For examples, seminal papers [1] [2] and [3] derived optimal execution strategies only in the stock exchange, which considered the trade-off between the market impact risk and the volatility (price change) risk. On the other hand, alternative trading platforms at the off-exchange referred to dark pools and internal crossing have been attached the attention. For more details refer to [4]. Since the invisibility of liquidity at the off-exchange trading does not impact on the price, that trading venues are popular platforms for the large trade. When the large trader adopts her executions at an off-exchange trading venue, especially, internal crossing with a broker, it is necessary to obtain some consents with the counter-party about the execution date (time), price, and volume. For example, the large trader executes with the broker (counter party) a shares at noon on specific day with the opening price of that day. Although it is also possible to make a contract using the closing price, which we call "closing price guaranteed execution", as mentioned in [5], the closing price guaranteed execution is misalignment because the broker is able to accumulate his holdings at the stock exchange with changing the price to be desirable as possible. That is, the broker could make a profit by round trip trade as in [6]. Thus, in theoretical and practical points of view, VWAP (Volume Weighted Average Price) is often used for an execution price at the off-exchange trading venue as in [7]. But it is difficult to treat the VWAP in a dynamic optimization problem. Thus, the VWAP guaranteed execution problem is often treated in a static framework.

In this paper, we derive an optimal execution strategy at the traditional trading and off-exchange trading venues where we adopt a closing price guaranteed execution (more accurately called after-hour-trading like ToSTNeT at Tokyo Stock Exchange). First of all, we establish the framework in which a single large trader makes a contract with a broker to execute her holdings at the specific intraday closing price before executing at the traditional stock exchange. That is, after the large trader executes at the traditional stock exchange within the day, she makes a trade of remaining volume with the broker at the closing price of that traditional stock exchange based on the contract. In this situation, the contract about the trading volume does not reach an agreement. However, using the approach of [8] that shows an optimal execution strategy making decisions dynamically is in the class of static ones, we derive the optimal allocations of both traditional stock exchange trading volume and off-exchange trading volume at the initial time. Therefore, we show that we can contain not only time and price but also the execution volume in this contract (agreement) at the off-exchange trading. However, since we cannot deny the possibility of the price manipulation, we will put the strong assumption about the broker.

This paper is organized as follows. A general stock price model is introduced on the specific stock exchange and we give an assumption on off-exchange trad- 
ing in Section 2. If we do not impose such an assumption then the large trader is always defeated in the broker, accordingly no agreement in over-the-counter trading would be reached. Section 3 presents the wealth process of a single large trader and derives the optimal execution strategy in both trading venues. This main idea enables the large trader to make a contract (agreement) with a broker to trade predetermined volume at the closing price before the intraday trading in the stock exchange. We also show that this problem has another explanation. In Section 4, numerical examples are represented, and Section 5 concludes this paper.

\section{Setup}

In this section, we define the stock price model in the exchange which is partially based on [8] and assume some conditions. We fix a probability space $(\Omega, \mathcal{F}, P)$ and assume that all stochastic processes are defined on this space with a filtration. In particular, the i.i.d. random sequence $\left\{\varepsilon_{t}\right\}$ represents the public news effect and follows as $\varepsilon_{t} \sim N\left(\mu_{\varepsilon}, \sigma_{\varepsilon}^{2}\right)$.

\subsection{Price Model in the Exchange Trading}

Let $p_{t}$ denote the stock price at time $t$ and $\hat{p}_{t}$ the execution price at time $t^{+}$. We assume that if the large trader submits the large volume, the stock price is immediately sifted up and her submitted orders are always executed entirely. That is, the order book with block shape is fully liquid for both the bid and ask sides but we define the depth of the book of this stock to be always $1 / \lambda_{t}$ for all price at time $t$, where $\lambda_{t} \in \mathbb{R}_{+}$represents the price change per unit execution. Then, if we define $q_{t}$ as the submitted order volume of the large trader then

$$
\hat{p}_{t}=p_{t}+\lambda_{t} q_{t} .
$$

Here, we define the price process as

$$
p_{t+1}=p_{t}+\lambda_{t} q_{t}\left\{\alpha_{t} \mathrm{e}^{-\rho}+\left(1-\alpha_{t}\right)\right\}-S_{t}+\varepsilon_{t+1},
$$

where $\alpha_{t} \in[0,1]$ is the rate of immediate return to previous price level, $\rho \in \mathbb{R}_{+}$ is the resilience speed, and

$$
S_{t}:=\mathrm{e}^{-\rho t}\left(1-\mathrm{e}^{-\rho}\right) \sum_{k=1}^{t-1} \lambda_{k} \mathrm{e}^{k} q_{k}
$$

represents the cumulative effects of the past large trades. On the other hand, concerning $p_{t}$, [8] did not consider the permanent price impact explicitly. The reason why we explicitly define the permanent effect is that, within the intraday large trading, we should consider the effect of the large trading continues all day long to some degree.

When $\rho \rightarrow \infty$, since the stock price reverts to immediately, we have $S_{t} \rightarrow 0$. Moreover, in another case when $\rho \rightarrow 0$, the stock price is not recovered to the previous level at all. Therefore, it is equivalent to $\alpha_{t}=0$. Actually, if $\alpha_{t}=0$ or $\rho \rightarrow 0$ then, from Equation (2), we have

$$
p_{t+1}=p_{t}+\lambda_{t} q_{t}+\varepsilon_{t+1} .
$$

Of course in this case, the pure price manipulation in the sense of [6] is ab- 
sence while the transaction-triggered-price manipulation in the sense of [9] may appear. Notice that if we do not consider the permanent impact, that is to say, if $\alpha_{t} \rightarrow 1$ then

$$
p_{t+1}=p_{t}+\lambda_{t} q_{t} \mathrm{e}^{-\rho}-S_{t}+\varepsilon_{t+1},
$$

which is same as [3], and if $\rho \rightarrow \infty$ then, since $S_{t} \rightarrow 0$, we have

$$
p_{t+1}=p_{t}+\left(1-\alpha_{t}\right) \lambda_{t} q_{t}+\varepsilon_{t+1}
$$

which is same as [10].

Here, transience of price impact means that this price impact will decay over time. One of the first models for transient impact was proposed by [3] for the case of exponential decay of price impact. The decay of price impact is modeled by means of a (typically non-increasing) function $G$, so called the decay kernel or resilience function. For more detail refer to [3] and [11].

\subsection{Assumptions in the Off-Exchange Trading}

In general, the large trader often seeks her liquidity at the off-exchange before executing in the stock exchange because of the market impact. In the case of considering the off-exchange trading, there is at least the need to clarify the timing and price of trade.

In this paper, we consider the off-exchange trading after the intraday execution at the stock exchange. Although it is something like the after hour trading, since we do not limit the exchange trading, we use the term off-exchange in a broad sense. We denote $T+1$ the closed time on the stock exchange and we suppose that the large trader seeks the liquidity until time $T$ at the stock exchange before the execution with a broker at the off-exchange. Although various prices at the off-exchange trading are taken into account, for examples, intraday VWAP, opening price, or closing price in that day, we consider the large trader could agree with the broker to execute at the closing price of that day at time $T+1$ or later.

However, there would be some opportunities for both the large trader and the broker to manipulate the price in such a trading without assuming any conditions. Therefore, we make the following assumption about the broker.

\section{Assumption}

At the off-exchange trading, the brokers can supply their holding infinitely. Thus, they do not demand their liquidity at the stock exchange.

Although this assumption seems to be unrealistic, this can be implemented by a legal regulation or a contractual restriction. If this assumption is absent, the large traders are always defeated by the brokers because by accumulating the holding of the brokers over the day while the stock price is sifted up, the broker can palm off on the large trader at the off-exchange trading for a ridiculously high price. For example, assume that a large trader made a contract with a broker to purchase 100,000 shares of the stock at the closing price after the stock exchange trading period and this contract was agreed before the exchange trading time. If the broker did not hold the stock of 100,000 shares, he is also able to 
purchase in that stock exchange. Because of his massive purchasing, this stock price is pulled up his desirable direction, especially the resilience of the price is suppressed before the closing time of the stock exchange, and he can sell his holding (100,000 shares) at a higher price. This is the pure price manipulation in the sense of [6].

On the other hand, from the point of view of the large trader, she also manipulates the market if some off-exchange trading is possible. However, since the purpose of this paper is focused on an analysis of the optimizing behavior of the large trader, we do not impose any constraint to the large trader. We will illustrate in Section 4 that the large trader would manipulates the price by a level of the cost at the off-exchange trading.

The problems without this assumption should be treated in the framework of the contract theory which is a rather new applied field of non-cooperative game theory. Since the studies on this perspective in which the trading strategy of the large trader might be affected by the strategic behavior of the broker and vice versa, is beyond the scope of this paper, we remain them for our future research topics.

\section{Optimal Execution Strategy}

In this section, we derive optimal execution strategy in the stock exchange and allocation to both trading venues. Therefore, we introduce more assumptions and setting additionally to those in Section 2. Suppose that the large trader slices her orders into $T$ pieces with equidistant time intervals. After the execution at the stock exchange, she trades her remaining volumes with the broker at the off-exchange with the price at time $T+1$, that is to say, the closing price. We assume that this off-exchange trading is guaranteed, but, for now, we do not specify the execution volume of the off-exchange trading at the contract time. Thus, the broker agrees with a counter-trade at the bidding of the volume of the large trader. Although this assumption seems to be strong, however, the following Theorem of Section 3.2 enables us to reduce this setting.

\subsection{Wealth Process and Objective Function of the Large Trader}

We consider the optimization problem of dynamic execution strategy in which the large trader maximizes her expected utility from the terminal wealth $w_{T+1}$. We define the wealth at time $T+1$, that is, after the off-exchange trading as

$$
w_{T+1}=w_{T}-\hat{p}_{T} q_{T}-p_{T+1} Q_{T+1}-C_{T+1} Q_{T+1}^{2},
$$

where $C_{T+1} Q_{T+1}^{2}$ is a cost term for trade with the broker which includes a transaction fee, costs of orders which have not been completely liquidated, and others as in [12], and $p_{T+1} Q_{T+1}$ represents the execution fee at the closing price.

At time $t(=1, \cdots, T)$ in the ordinary exchange trading, we have

$$
w_{t+1}=w_{t}-\hat{p}_{t} q_{t} .
$$

Now, we define the expected utility of the large trader under the trading strategy $\pi$ by a negative exponential (CARA type) von-Neumann and Mor- 
genstern (vN-M) utility function as in [8]:

$$
V_{t}^{\pi}=E_{t}^{\pi}\left[-\exp \left\{-R w_{T+1}\right\} \mid w_{t}, p_{t}, Q_{t}, S_{t}, q_{t}\right]
$$

where $R$ is the risk aversion parameter and we utilize the fact that the 4-tuple $\left(w_{t}, p_{t}, Q_{t}, S_{t}\right)$ becomes a state or sufficient statistics of the decision process of the trader.

Further, we define the optimal value function as

$$
V_{t}:=\operatorname{esssup} V_{t}^{\pi} .
$$

By the principle of optimality, the optimality equation (Bellman equation) becomes as

$$
V_{t}\left(w_{t}, p_{t}, Q_{t}, S_{t}\right)=\sup _{q_{t} \in \mathbb{R}} E\left[V_{t+1}\left(w_{t+1}, p_{t+1}, Q_{t+1}, S_{t+1}\right) \mid w_{t}, p_{t}, Q_{t}, S_{t}, q_{t}\right] .
$$

\subsection{Main Results}

\section{Theorem}

Suppose that i.i.d. random variables $\varepsilon_{t}(t=1, \cdots, T+1)$ follow normal distributions and the risk-averse large trader has CARA type vN-M utility. If $C_{T+1}$ is deterministic then a static execution strategy becomes optimal.

Proof:

Using the inductive method, we derive the optimal execution volumes backwardly from time $T+1$. For simplicity, we set

$$
\alpha_{t} \mathrm{e}^{-\rho}+\left(1-\alpha_{t}\right)=: m_{t} .
$$

Firstly, at time $T+1$, since the remaining whole volumes of the large trader are executed at the closing price, we set

$$
q_{T+1}=Q_{T+1} .
$$

Secondly, at time $T$,

$$
V_{T}\left(w_{T}, p_{T}, Q_{T}, S_{T}\right)=\sup _{q_{T} \in \mathbb{R}} E\left[V_{T+1}\left(w_{T+1}, p_{T+1}, Q_{T+1}, S_{T+1}\right) \mid w_{T}, p_{T}, Q_{T}, S_{T}, q_{T}\right] .
$$

Since

$$
\begin{aligned}
E & {\left[V_{T+1}\left(w_{T+1}, p_{T+1}, Q_{T+1}, S_{T+1}\right) \mid w_{T}, p_{T}, Q_{T}, S_{T}, q_{T}\right] } \\
= & -\exp \left\{-R w_{T}+R p_{T} Q_{T}+R A_{T}^{1} q_{T}^{2}-R\left(A_{T}^{2} Q_{T}-A_{T}^{3} S_{T}+A_{T}^{4}\right) q_{T}\right. \\
& \left.-R\left(S_{T}-\mu_{\varepsilon}\right) Q_{T}+R\left(C_{T+1}+\frac{R}{2} \sigma_{\varepsilon}^{2}\right) Q_{T}^{2}\right\},
\end{aligned}
$$

the optimal execution volume at time $T$ is

$$
q_{T}^{*}=\frac{A_{T}^{2} Q_{T}-A_{T}^{3} S_{T}+A_{T}^{4}}{2 A_{T}^{1}}=\frac{\left(-\lambda_{T} m_{T}+2 C_{T+1}+R \sigma_{\varepsilon}^{2}\right) Q_{T}-S_{T}+\mu_{\varepsilon}}{2 \lambda_{t}\left(1-m_{T}\right)+2 C_{T+1}+R \sigma_{\varepsilon}^{2}},
$$

where

$$
\begin{aligned}
A_{T}^{1} & :=\lambda_{T}\left(1-m_{T}\right)+C_{T+1}+\frac{R}{2} \sigma_{\varepsilon}^{2}, \\
A_{T}^{2} & :=-\lambda_{T} m_{T}+2 C_{T+1}+R \sigma_{\varepsilon}^{2},
\end{aligned}
$$




$$
A_{T}^{3}:=1,
$$

$$
A_{T}^{4}:=\mu_{\varepsilon} .
$$

Then, the optimal value function becomes

$$
\begin{aligned}
& V_{T}\left(w_{T}, p_{T}, Q_{T}, S_{T}\right) \\
& =-\exp \left\{-R w_{T}+R p_{T} Q_{T}-R \frac{\left(A_{T}^{2} Q_{T}-A_{T}^{3} S_{T}+A_{T}^{4}\right)^{2}}{4 A_{T}^{1}}-R\left(S_{T}-\mu_{\varepsilon}\right) Q_{T}+R\left(C_{T+1}+\frac{R}{2} \sigma_{\varepsilon}^{2}\right) Q_{T}^{2}\right\} \\
& =-\exp \left\{-R w_{T}+R p_{T} Q_{T}\right\} \exp \left\{R\left(B_{T}^{1} Q_{T}^{2}+B_{T}^{2} Q_{T}+B_{T}^{3} Q_{T} S_{T}+B_{T}^{4} S_{T}^{2}+B_{T}^{5} S_{T}+B_{T}^{6}\right)\right\}, \\
& \text { where } \\
& B_{T}^{1}:=-\frac{\left(A_{T}^{2}\right)^{2}}{4 A_{T}^{1}}+C_{T+1}+\frac{R}{2} \sigma_{\varepsilon}^{2}, \\
& B_{T}^{2}:=-\frac{A_{T}^{2} A_{T}^{4}}{2 A_{T}^{1}}+\mu_{\varepsilon}, \\
& B_{T}^{3}:=\frac{A_{T}^{2} A_{T}^{3}}{2 A_{T}^{1}}-1, \\
& B_{T}^{4}:=-\frac{\left(A_{T}^{3}\right)^{2}}{4 A_{T}^{1}}, \\
& B_{T}^{5}:=\frac{A_{T}^{3} A_{T}^{4}}{2 A_{T}^{1}}, \\
& B_{T}^{6}:=-\frac{\left(A_{T}^{4}\right)^{2}}{4 A_{T}^{1}} .
\end{aligned}
$$

Similarly, at time $t$, by calculating inductively, we get

$$
q_{t}^{*}=\frac{A_{t}^{2} Q_{t}-A_{t}^{3} S_{t}+A_{t}^{4}}{2 A_{t}^{1}},
$$

where

$$
\begin{aligned}
A_{t}^{1} & :=\lambda_{t}\left(1-m_{t}\right)+B_{t+1}^{1}-l_{t}\left(B_{t+1}^{3}-B_{t+1}^{4} l_{t}\right)+\frac{R}{2} \sigma_{\varepsilon}^{2}, \\
A_{t}^{2} & :=-\lambda_{t} m_{t}+2 B_{t+1}^{1}-B_{t+1}^{3} l_{t}+R \sigma_{\varepsilon}^{2}, \\
A_{t}^{3} & :=-B_{t+1}^{3} \mathrm{e}^{-\rho}+2 B_{t+1}^{4} l_{t} \mathrm{e}^{-\rho}+1, \\
A_{t}^{4} & :=B_{t+1}^{2}-B_{t+1}^{5} l_{t}+\mu_{\varepsilon} .
\end{aligned}
$$

Thus, the optimal value function is reduced to

$$
\begin{aligned}
& V_{t}\left(w_{t}, p_{t}, Q_{t}, S_{t}\right) \\
& =-\exp \left\{-R w_{t}+R p_{t} Q_{t}\right\} \exp \left\{R\left(B_{t}^{1} Q_{t}^{2}+B_{t}^{2} Q_{t}+B_{t}^{3} Q_{t} S_{t}+B_{t}^{4} S_{t}^{2}+B_{t}^{5} S_{t}+B_{t}^{6}\right)\right\},
\end{aligned}
$$

where

$$
B_{t}^{1}:=-\frac{\left(A_{t}^{2}\right)^{2}}{4 A_{t}^{1}}+B_{t+1}^{1}+\frac{R}{2} \sigma_{\varepsilon}^{2},
$$




$$
\begin{aligned}
B_{t}^{2} & :=-\frac{A_{t}^{2} A_{t}^{4}}{2 A_{t}^{1}}+B_{t+1}^{2}+\mu_{\varepsilon}, \\
B_{t}^{3} & :=\frac{A_{t}^{2} A_{t}^{3}}{2 A_{t}^{1}}+B_{t+1}^{3} \mathrm{e}^{-\rho}-1, \\
B_{t}^{4} & :=-\frac{\left(A_{t}^{3}\right)^{2}}{4 A_{t}^{1}}+B_{t+1}^{4} \mathrm{e}^{-2 \rho}, \\
B_{t}^{5} & :=\frac{A_{t}^{3} A_{t}^{4}}{2 A_{t}^{1}}+B_{t+1}^{5} \mathrm{e}^{-\rho}, \\
B_{t}^{6} & :=-\frac{\left(A_{t}^{4}\right)^{2}}{4 A_{t}^{1}}+B_{t+1}^{6} .
\end{aligned}
$$

Since $A_{t}^{*}$ and $B_{t}^{*}$ are both deterministic, we conclude that the strategy $\pi=\left\{q_{t}^{*}\right\}$ is a static strategy.

According to the above theorem, it is possible for the large trader to control the volume of the guaranteed execution at the closing price before her execution at the stock exchange. Since this theorem guarantees the closing price guaranteed execution, the agreement that the large trader executes the predetermined volume with the broker beforehand could be achieved under the condition of the market and the level of $C_{T+1}$. But if the large trader can notice that the broker attempt to manipulate the price at the stock exchange after the agreement, the cost $C_{T+1}$ becomes higher, then she may not be interested in the contract with the broker.

\section{Proposition}

If $C_{T+1} \rightarrow \infty$ then the large trader does not use the off-exchange trading.

Proof:

From Equation (16), if $C_{T+1} \rightarrow \infty$ then

$$
q_{T}^{*}=\frac{A_{T}^{2} Q_{T}-A_{T}^{3} S_{T}+A_{T}^{4}}{2 A_{T}^{1}}=\frac{\left(-\lambda_{T} m_{T}+2 C_{T+1}+R \sigma_{\varepsilon}^{2}\right) Q_{T}-S_{T}+\mu_{\varepsilon}}{2 \lambda_{t}\left(1-m_{T}\right)+2 C_{T+1}+R \sigma_{\varepsilon}^{2}} \rightarrow Q_{T} .
$$

Therefore, $Q_{T+1}=Q_{T}-q_{T}^{*} \rightarrow 0$. This means that the large trader completes her execution only in the stock exchange.

\subsection{Other Perspectives: The Case of Only Exchange Trading}

Here, we set that the large trader submits her orders only at the stock exchange on the intraday trading. Also, we define that

$$
C_{T+1}^{\prime}=p_{T+1}+C_{T+1} Q_{T+1} .
$$

In this case, we can regard $C_{T+1}^{\prime}$ as the cost under which the large trader put off her holding the next day or later. If the large trader is able to make an estimate of this latent cost, she can manage how much volumes she executes in the exchange trading at that day and holds over next day or later. The optimization problem from this point of view is an extension of [8] in which the large trader must execute her whole holdings in the planning horizon. But in the real situation, such strong constraint seems to be rare, and the large trader would often 
hold over her executions if she expects the market condition of the next day is better than that of today to some extent. The above proposition also indicates that if $C_{T+1} \rightarrow \infty$, that is equivalently, if $C_{T+1}^{\prime} \rightarrow \infty$, then the large trader do not leave her holdings to the next day or later.

\section{Comparative Statics}

In this section, we illustrate some numerical examples of execution volume in both the stock exchange and off-exchange within the day. The large trader makes a contract to execute the predetermined volume with the broker at the off-exchange trading venue before she executes at the stock exchange. We set $T=13$ periods which indicates the trading at the stock exchange, and $T+1=14$ at the off-exchange. For simplicity, we assume $\mu_{\varepsilon}=0$, then, from Equation (21) \& Equation (22), $A_{t}^{4}=B_{t}^{2}=B_{t}^{5}=B_{t}^{6}=0$. Moreover, we fix the parameters as $\lambda_{t}=0.001, \quad \alpha_{t}=0.5, \rho=0.1, \quad \sigma_{\varepsilon}^{2}=0.02, C_{T+1}=0.01$, $R=0.001$, and $\bar{Q}=100,000$.

Figure 1 illustrates the risk aversion effects on the execution at both trading venues. As the more risk averse the large trader is, the faster she executes at the exchange and the smaller amounts she executes at the off-exchange. Table 1 shows the off-exchange trading (at $t=T+1=14$ ) volume. This represents that the risk averse large trader considers more price change (volatility) risk because of closing price guaranteed execution.

Figure 2 shows that the large trader has a possibility to manipulate the market by the level of $C_{T+1}$. If the level of the cost $C_{T+1}$ is lower than the price (market)

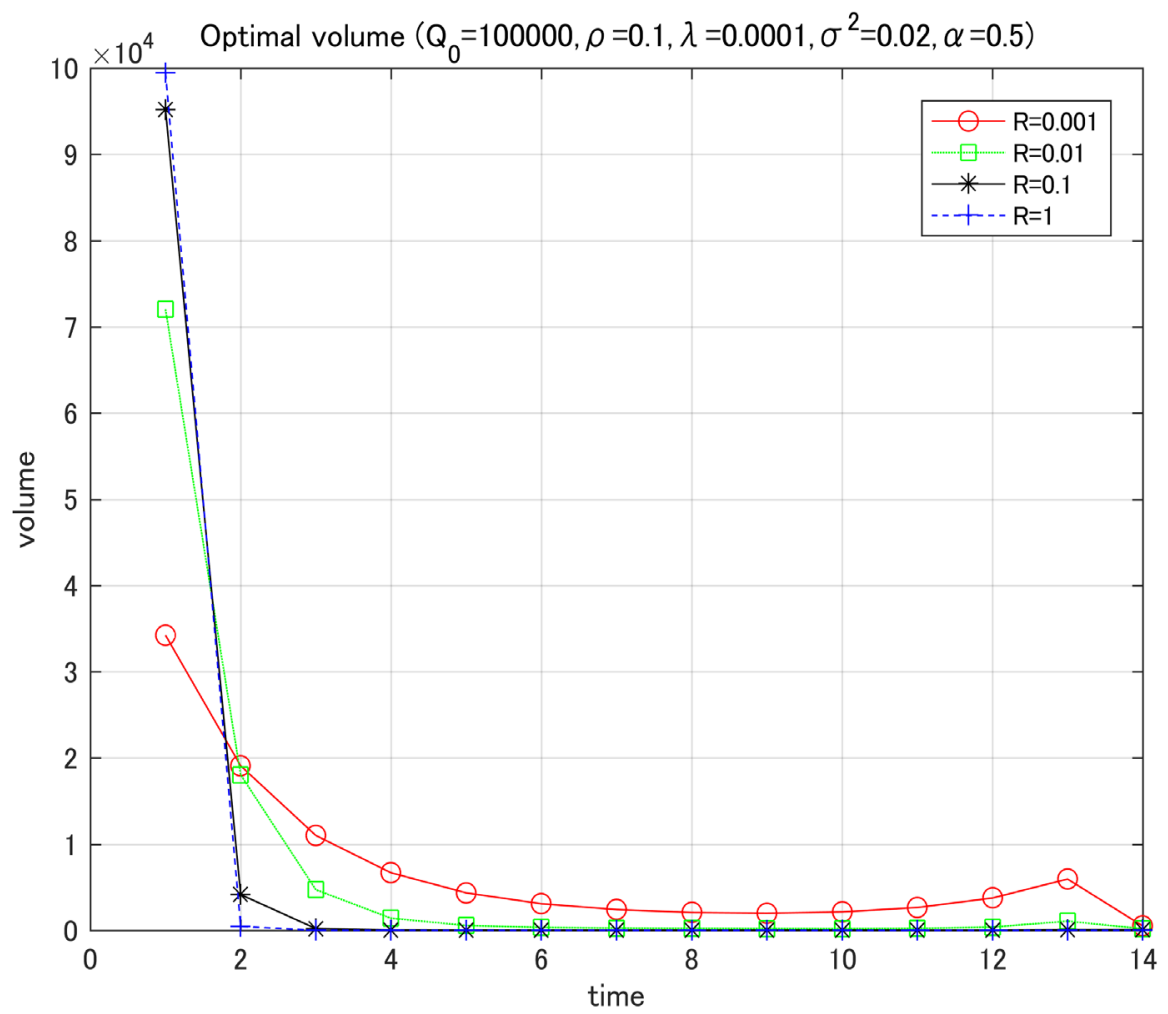

Figure 1. Optimal execution in terms of risk aversion coefficient $R$. 
Table 1. Submission order volume at off-exchange trading venue.

\begin{tabular}{ccccc}
\hline Risk aversion & $R=0.001$ & $R=0.01$ & $R=0.1$ & $R=1$ \\
\hline Off-exchange trading volume & 512 & 197 & 76 & 13 \\
\hline
\end{tabular}

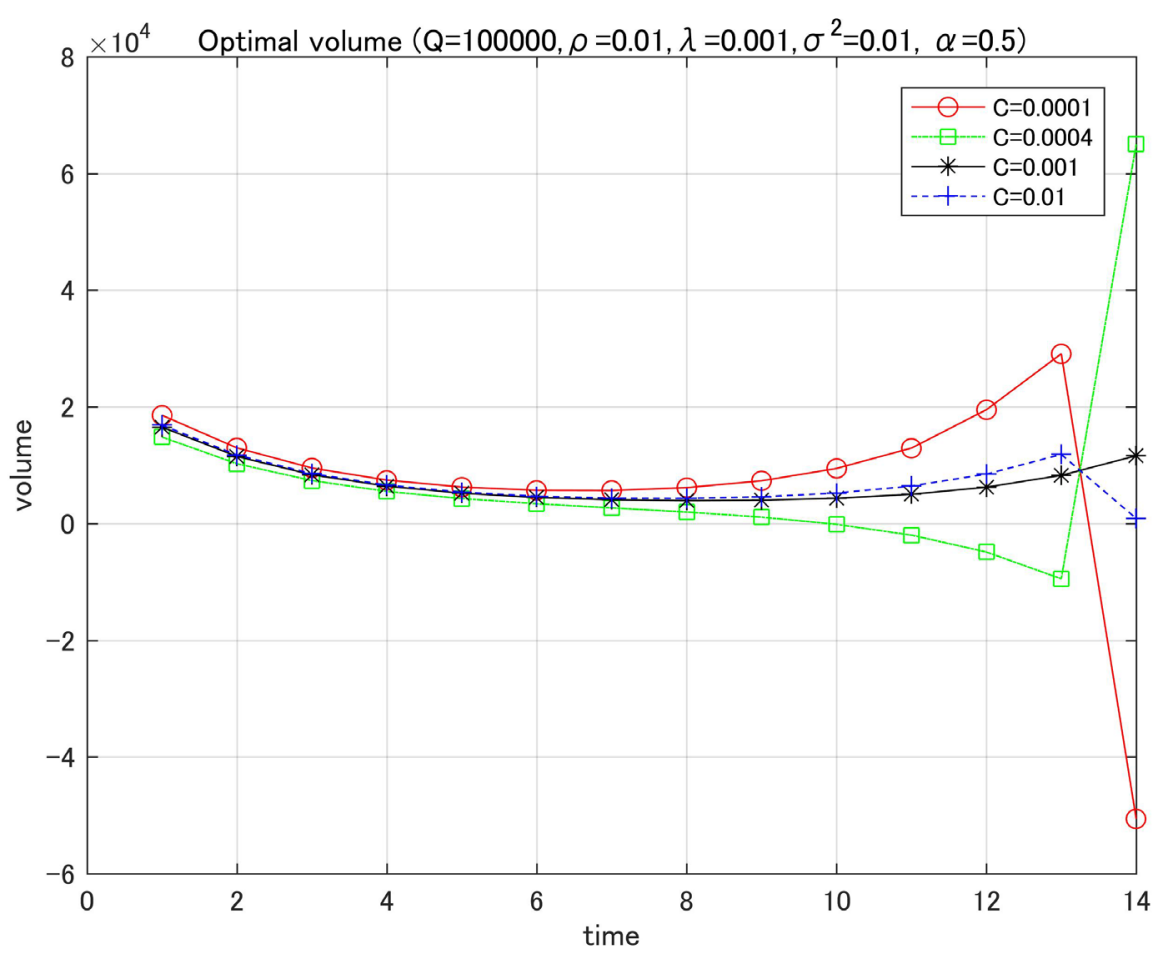

Figure 2. Optimal execution in terms of cost $C$.

impact term $\lambda_{t}=0.001$, the large trader leads the price to her desired direction intentionally. In this numerical case, the sign of the off-exchange trading changes within the range of $0.000269820<C_{T+1}<0.000269821$. If

$C_{T+1}<0.000269820$, the large trader would make a contract with the broker to sell in spite of planning to purchase. Since she certainly sells her holdings at the closing price based on the agreement, she purchases so that the closing price gets higher considering the volatility risk.

\section{Conclusion}

In this paper we derived the optimal execution strategy for a large trader who affects the stock price considering both the traditional stock exchange and off-exchange trading venues. We set the large trader makes a contract with a broker to be guaranteed her execution at the closing price on the intraday trading before she submits her order to the exchange. Then we showed that the large trader could control her order volumes before executing at the traditional stock exchange. However, without the requirement for the broker not to manipulate the stock price, since the large trader can perceive to suffer a loss, she does not make a contract with the broker at the off-exchange trading venue. On the other hand, when the large trader can estimate that the cost of off-exchange trading is lower than the market impact cost, she also manipulates the market. Moreover, 
we indicated another point of view whether to carry over the execution of the large trader to the next day or not. Similarly, in this case, we showed that the carryover of the execution depends on the market impact cost level at the trading day. Concerning more realistic but more complicated cases about the price manipulation of the large trader, game theoretical analysis of the VWAP guaranteed execution and the effects of the broker's strategic behavior is left for our future research.

\section{Acknowledgements}

We thank an anonymous referee and the editorial staffs for their constructive comments.

\section{References}

[1] Almgren, R. and Chriss, N. (2000) Optimal Execution of Portfolio Transactions. Journal of Risk, 3, 5-39. https://doi.org/10.21314/JOR.2001.041

[2] Bertsimas, D. and Lo, A. (1998) Optimal Control of Execution Costs. Journal of Financial Markets, 1, 1-50. https://doi.org/10.1016/S1386-4181(97)00012-8

[3] Obizhaeva, A. and Wang, J. (2013) Optimal Trading Strategy and Supply/Demand Dynamics. Journal of Financial Markets, 16, 1-32. https://doi.org/10.1016/j.finmar.2012.09.001

[4] Mittal, H. (2008) Are You Playing in a Toxic Dark Pool? A Guide to Preventing Information Leakage. Journal of Trading, 3, 20-33. https://doi.org/10.3905/jot.2008.708833

[5] Busseti, E. and Boyd, S. (2015) Volume Weighted Average Price Optimal Execution. arXiv:1509.08503v1

[6] Huberman, G. and Stanzl, W. (2004) Price Manipulation and Quasi-Arbitrage. Econometrica, 74, 1247-1275. https://doi.org/10.1111/j.1468-0262.2004.00531.x

[7] Guéant, O. and Royer, G. (2014) VWAP Execution and Guaranteed VWAP. SIAM Journal on Financial Mathematics, 5, 445-471. https://doi.org/10.1137/130924676

[8] Kuno, S. and Ohnishi, M. (2015) Optimal Execution in Illiquid Market with the Absence of Price Manipulation. Journal of Mathematical Finance, 5, 1-14. https://doi.org/10.4236/jmf.2015.51001

[9] Alfonsi, A., Schied, A. and Slynko, A. (2012) Order Book Resilience, Price Manipulation, and the Positive Portfolio Problem. SIAM Journal on Financial Mathematics, 3, 511-533. https://doi.org/10.1137/110822098

[10] Huberman, G. and Stanzl, W. (2005) Optimal Liquidity Trading. Review of Finance, 9, 165-200. https://doi.org/10.1007/s10679-005-7591-5

[11] Gatheral, J. (2010) No-Dynamic-Arbitrage and Market Impact. Quantitative Finance, 10, 749-759. https://doi.org/10.1080/14697680903373692

[12] Cartea, A. and Jaimungal, S. (2016) A Closed-Form Execution Strategy to Target Volume Weighted Average Price. SIAM Journal on Financial Mathematics, 7, 760 785. http://dx.doi.org/10.1137/16M1058406 